\title{
A Novel Approach to Identifying the Cosine Similarity using TF-IDF
}

\author{
Gottapu Mohana Rao ${ }^{1}$, Dr. P. Satheesh ${ }^{2}$, Dr. B. Srinivas ${ }^{3}$ \\ M.Tech, Department of CSE, MVGR College of Engineering, Vizianagaram, India ${ }^{1}$ \\ Associate Professor, CSE Department, MVGR College of Engineering, Vizianagaram, India ${ }^{2,3}$
}

\begin{abstract}
An expanding number of database applications today require modern inexact string coordinating abilities. Cases of such application ranges incorporate information coordination and information cleaning. Cosine closeness has turned out to be a strong metric for scoring the comparability between two strings, and it is progressively being utilized as a part of complex questions. A quick test confronted by current database analyzers is to discover precise and productive techniques for evaluating the selectivity of cosine comparability predicates. To the best of our insight, there are no known techniques for this issue. In this paper, we display the principal approach for assessing the selectivity of TF-IDF based cosine likeness predicates. We assess our approach on three diverse genuine datasets and demonstrate that our technique regularly delivers gauges that are inside $40 \%$ of the real selectivity. The cosine likeness is a measure of similitude between two nonzero vectors of an inward item space. This cosine comparability can be utilized to look at the string from the given archives, the term recurrence is utilized to think about the given string from the diverse reports we have. The converse report recurrence is to discover significant archives coordinating the question.
\end{abstract}

Keywords: Cosine Similarity, Term Frequency, Inverse Document Frequency.

\section{INTRODUCTION}

Cosine Similarity:-

Cosine Similarity is a measure of similitude between two nonzero vectors of an inward item space that measures the cosine of the point between them. From each archive we infer a vector.

The arrangement of reports in an accumulation at that point is seen as an arrangement of vectors in a vector space. Each term will have its own hub. Utilizing the recipe given beneath we can discover the similitude between any two archives. Cosine Similarity $(\mathrm{d} 1, \mathrm{~d} 2)=$ Dot item $(\mathrm{d} 1, \mathrm{~d} 2) /\|\mathrm{d} 1\| *\|\mathrm{~d} 2\|$

Speck item $(\mathrm{d} 1, \mathrm{~d} 2)=\mathrm{d} 1[0] * \mathrm{~d} 2[0]+\mathrm{d} 1[1] * \mathrm{~d} 2[1] * \ldots * \mathrm{~d} 1[\mathrm{n}] * \mathrm{~d} 2[\mathrm{n}]$

$\|\mathrm{d} 1\|=$ square root $(\mathrm{d} 1[0] 2+\mathrm{d} 1[1] 2+\ldots+\mathrm{d} 1[\mathrm{n}] 2)$

$\|\mathrm{d} 2\|=$ square $\operatorname{root}(\mathrm{d} 2[0] 2+\mathrm{d} 2[1] 2+\ldots+\mathrm{d} 2[\mathrm{n}] 2)$

\section{Term Frequency:-}

In actuality each archive will be of various size. On a vast archive the recurrence of the terms will be significantly higher than the littler ones. Henceforth we have to standardize the report in light of its size. A basic trap is to isolate the term recurrence by the aggregate number of terms.

\section{Inverse Document Frequency:-}

The primary reason for doing a pursuit is to discover applicable archives coordinating the question. In the initial step all terms are considered similarly critical. Truth be told sure terms that happen too often have little power in deciding the significance. We require an approach to burden the impacts of too regularly happening terms. Likewise the terms that happen less in the archive can be more applicable. We require an approach to weigh up the impacts of less much of the time happening terms logarithms causes us to take care of this issue.

\section{RELATED WORK}

Cosine likeness is a vector-based measure of the similitude of two strings. The essential thought behind cosine closeness is to change each string into a vector in some high dimensional space with the end goal that comparable strings are near each other. The cosine of the edge between two vectors is a measure of how "comparative" they are, which thusly, is a measure of the comparability of these strings. In the event that the vectors are of unit length, the cosine of the point between them is basically the dab result of the vectors. There are numerous methods for changing a string in the database into a vector. The TF-IDF vector is a prevalent decision for this portrayal. The TF-IDF vector is made out of the result of a term recurrence and the backwards record recurrence for every token that shows up in the string. The way toward developing the TF-IDF vector is depicted beneath. 
As an initial move towards executing the cosine similitude predicate, we develop a TF-IDF vector for each line in the connection. In the event that there are various string characteristics of enthusiasm for each column, at that point we have to figure a vector for each string quality. To keep the dialog straightforward, we will accept there is just a single string property in the connection that is utilized as a part of a cosine comparability operation. At the point when an inquiry comes in, the standardized TF-IDF vector comparing to the question is developed. The IDF of each term in the inquiry is only 1 . We register the speck result of this vector with the vector for each column in the database: this is the cosine comparability. In the event that the inquiry and the string share more terms, the speck item is higher.

What's more, in the event that they share more "extraordinary" terms, that adds to the score more

\section{EVALUATION}

STEP 1: Term Frequency Example:-

Here we have the following number of documents

Document 1: The game of life is a game of everlasting learning

Document 2: The unexamined life is not worth living

Document 3: Never stop learning

Let us imagine that you are doing a search on these documents with the following query: life learning

The query is a free text query. It means a query in which the terms of the query are typed freeform into the search interface, without any connecting search operators.

Table 1 TF for Document1,2,3:

TF for Document 1
\begin{tabular}{|l|l|l|l|l|l|l|l|l|}
\hline Document1 & the & game & of & life & is & a & everlasting & learning \\
\hline $\begin{array}{l}\text { Term } \\
\text { Frequency }\end{array}$ & 1 & 2 & 2 & 1 & 1 & 1 & 1 & 1 \\
\hline
\end{tabular}

TF for Document 2

\begin{tabular}{|l|l|l|l|l|l|l|l|}
\hline Document2 & the & unexamined & life & is & not & worth & living \\
\hline Term Frequency & 1 & 1 & 1 & 1 & 1 & 1 & 1 \\
\hline
\end{tabular}

TF for Document 3

\begin{tabular}{|l|l|l|l|}
\hline Document3 & never & stop & learning \\
\hline
\end{tabular}

the term game occurs two times. The total number of terms in the document is $\mathbf{1 0}$. Hence the normalized term frequency is $\mathbf{2} / \mathbf{1 0}=\mathbf{0 . 2}$. Given below are the normalized term frequencies for all the documents.

Table 2 Normalized TF for Document1,2:

\begin{tabular}{|c|c|c|c|c|c|c|c|c|}
\hline Document1 & the & game & of & life & is & a & everlasting & learning \\
\hline $\begin{array}{l}\text { Normalized } \\
\text { TF }\end{array}$ & 0.1 & 0.2 & 0.2 & 0.1 & 0.1 & 0.1 & 0.1 & 0.1 \\
\hline \multicolumn{9}{|c|}{ Normalized TF for Document 2} \\
\hline Documen & the & unexamin & & fe & is & not & worth & living \\
\hline
\end{tabular}

\section{STEP 2: IDF Example:-}

Let us compute IDF for the term game

IDF (game) $=1+\operatorname{loge}($ Total Number Of Documents / Number Of Documents with term game is)

There are 3 documents in all $=$ Document 1 , Document2, Document 3 The term game appears in Document 1

$\operatorname{IDF}($ game $)=1+\operatorname{loge}(3 / 1)$

$1+1.098726209$

$=\quad 2.098726209$ 
Given below is the IDF for terms occurring in all the documents. Since the terms: the, life, is, learning occurs in 2 out of 3 documents they have a lower score compared to the other terms that appear in only one document.

\section{STEP 3: TF*IDF Example:-}

Recall that we are attempting to discover important archives for the inquiry: life learning

For each term in the inquiry increase its standardized term recurrence with its IDF on each archive. In Document1 for the term life the standardized term recurrence is 0.1 and its IDF is 1.405507153 . Duplicating them together we get $0.140550715(0.1 * 1.405507153)$. Given underneath is $\mathrm{TF} * \mathrm{IDF}$ figuring forever and learning in every one of the records.

Table 3 TF*IDF:

[

\begin{tabular}{|l|l|l|l}
\hline & Document1 & Document2 & Document3 \\
\hline life & 0.140550715 & 0.200786736 & 0 \\
\hline learning & 0.140550715 & 0 & 0.468502384 \\
\hline
\end{tabular}

Step 4: Vector Space Model - Cosine Similarity:.

Table 4 Vector space model:

\begin{tabular}{|l|l|}
\hline Terms & IDF \\
\hline The & 1.405507153 \\
\hline Game & 2.098726209 \\
\hline Of & 2.098726209 \\
\hline Life & 1.405507153 \\
\hline is & 1.405507153 \\
\hline A & 2.098726209 \\
\hline Everlasting & 2.098726209 \\
\hline Learning & 1.405507153 \\
\hline Unexamined & 2.098726209 \\
\hline Not & 2.098726209 \\
\hline worth & 2.098726209 \\
\hline living & 2.098726209 \\
\hline never & 2.098726209 \\
\hline stop & 2.098726209 \\
\hline
\end{tabular}

Cosine Similarity $(\mathrm{d} 1, \mathrm{~d} 2)=$ Dot product $(\mathrm{d} 1, \mathrm{~d} 2) /\|\mathrm{d} 1\| *\|\mathrm{~d} 2\|$

Dot product $(\mathrm{d} 1, \mathrm{~d} 2)=\mathrm{d} 1[0] * \mathrm{~d} 2[0]+\mathrm{d} 1[1] * \mathrm{~d} 2[1] * \ldots * \mathrm{~d} 1[\mathrm{n}] * \mathrm{~d} 2[\mathrm{n}]$

$\|\mathrm{d} 1\|=$ square root $\left(\mathrm{d} 1[0]^{2}+\mathrm{d} 1[1]^{2}+\ldots+\mathrm{d} 1[\mathrm{n}]^{2}\right)$

$\|\mathrm{d} 2\|=$ square root $\left(\mathrm{d} 2[0]^{2}+\mathrm{d} 2[1]^{2}+\ldots+\mathrm{d} 2[\mathrm{n}]^{2}\right)$

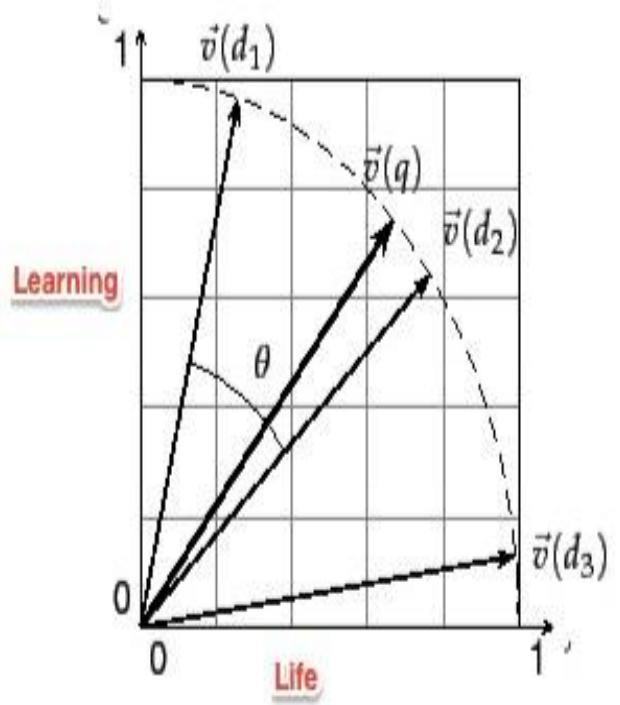

Figure 1 variation between life learning 
Vectors bargains just with numbers. In this case we are managing content archives. This was the motivation behind why we utilized TF and IDF to change over content into numbers with the goal that it can be spoken to by a vector.

The cosine measure comparability is another likeness metric that relies on upon imagining client inclinations as focuses in space. Hold as a main priority the picture of client inclinations as focuses in a $\mathrm{n}$-dimensional space. Presently envision two lines from the root, or point $(0,0, \ldots, 0)$, to each of these two focuses. At the point when two clients are comparable, they'll have comparable appraisals, thus will be moderately close in space at any rate, they'll be in generally a similar heading from the source. The point shaped between these two lines will be generally little. Conversely, when the two clients are disparate, their focuses will be inaccessible, and likely in various headings from the beginning, shaping a wide point. This edge can be utilized as the reason for a likeness metric similarly that the Euclidean separation was utilized to frame a similitude metric. For this situation, the cosine of the point prompts a closeness esteem. In case you're corroded on trigonometry, all you have to make sure to comprehend this is the cosine esteem is dependably amongst -1 and 1: the cosine of a little edge is almost 1 , and the cosine of a huge edge almost 180 degrees is near -1 . This is great, since little points ought to guide to high comparability, close to 1 , and extensive edges ought to guide to close -1 . The inquiry entered by the client can likewise be spoken to as a vector. We will Give us now a chance to figure the cosine closeness of the question and Document1. You can do the computation utilizing this instrument.

Cosine Similarity $($ Query,Document1 $)=$ Dot product $($ Query, Document1) $/ \|$ Query $\|*\|$ Document1 $\|$

Spot product(Query, Document1)

$=((0.702753576) *(0.140550715)+(0.702753576) *(0.140550715))$

$=0.197545035151$

$\|$ Query $\|=\operatorname{sqrt}((0.702753576) 2+(0.702753576) 2)=0.993843638185$

$\|$ Document $1 \|=\operatorname{sqrt}((0.140550715) 2+(0.140550715) 2)=0.198768727354$

Cosine Similarity $($ Query, Document $)=0.197545035151 /(0.993843638185) *(0.198768727354)$

$=0.197545035151 / 0.197545035151$

$=1$

Given beneath is the similitude scores for every one of the reports and the inquiry

Table 5 Cosine similarity between Document 1,2,3:

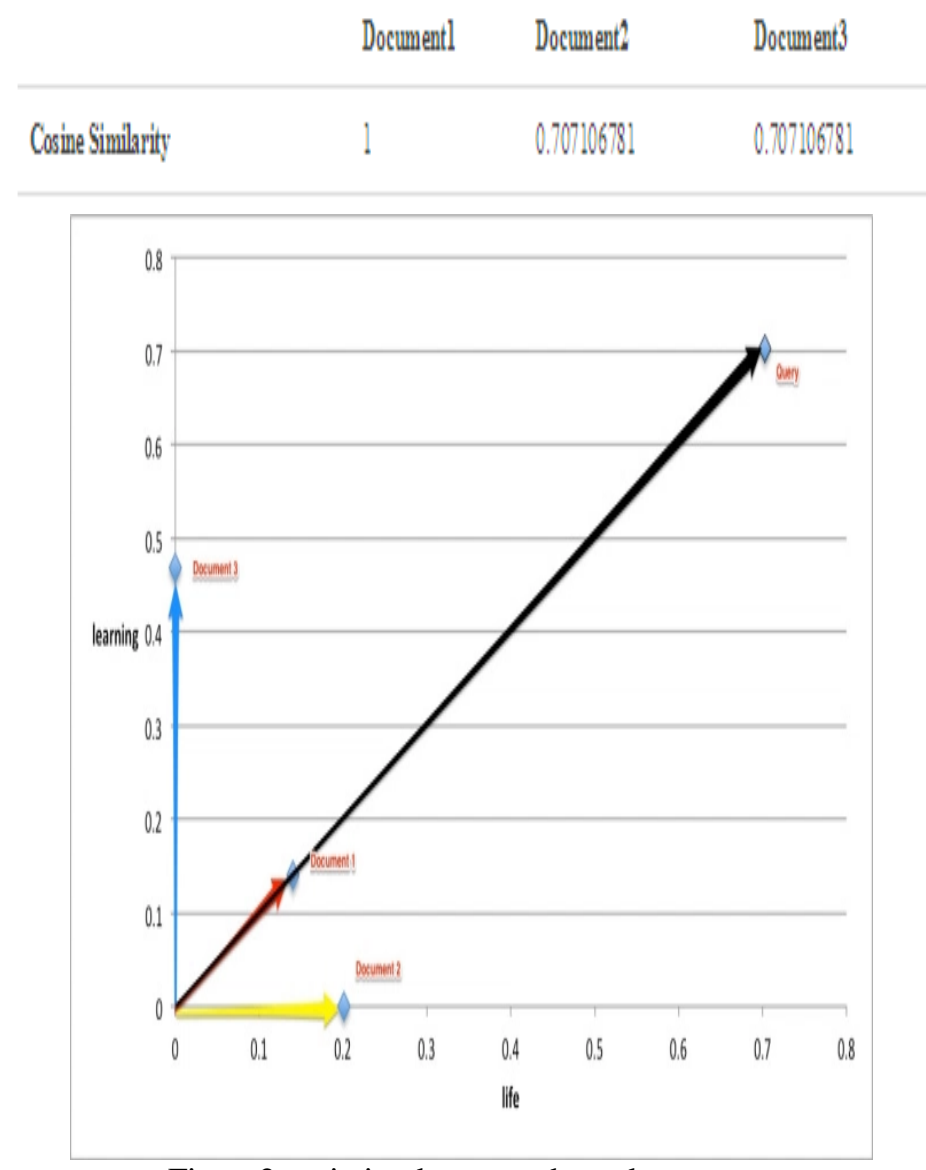

Figure2 variation between three documents 


\section{RESULTS}

Here we are using java language to implement the cosine similarity using TF-IDF, the results are displayed using the comparisons of each and every page other page.
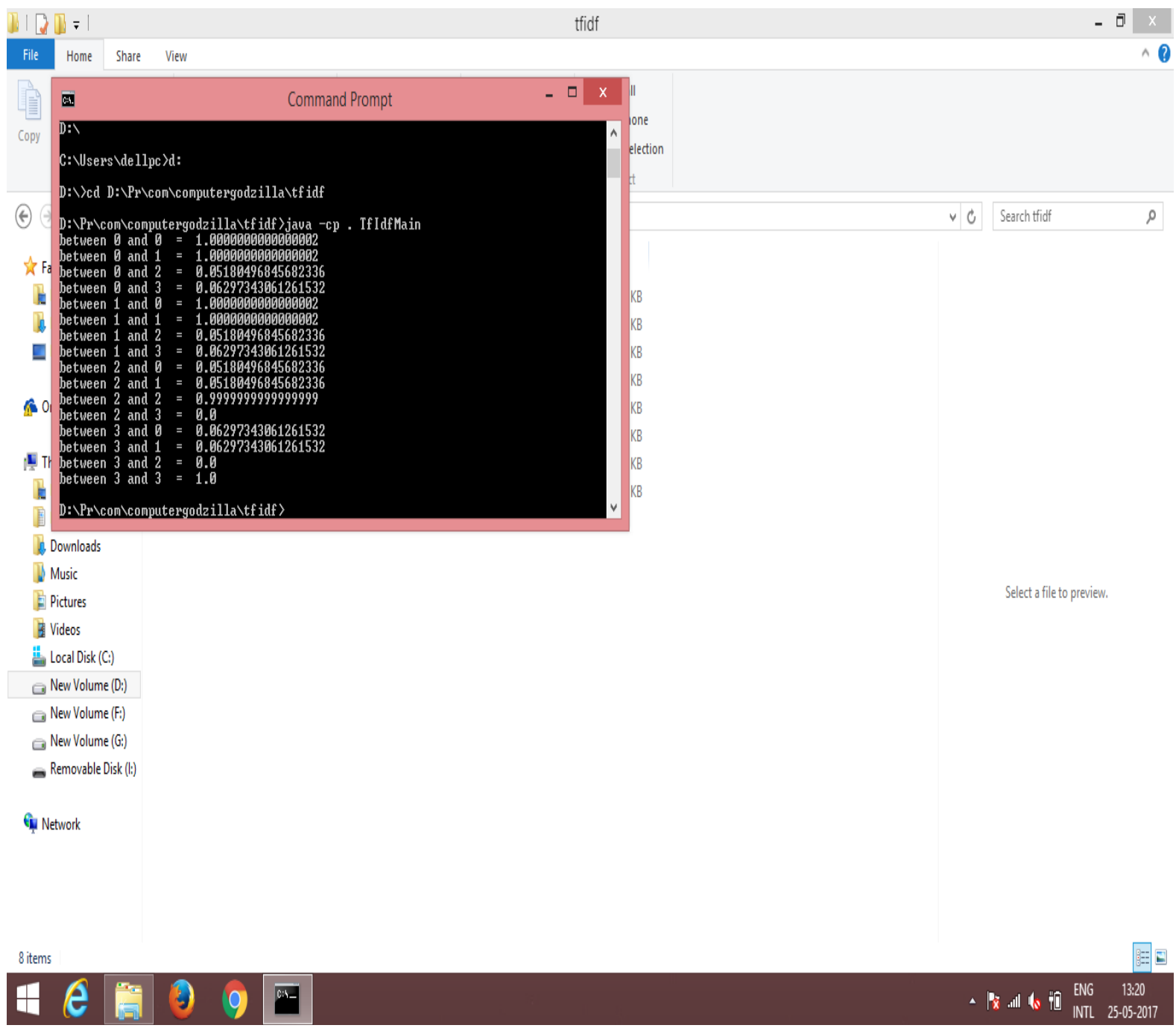

\section{CONCLUSION}

In this paper, we have presented the problem of estimating the selectivity of cosine similarity predicates. Here we are finding the similarity for term frequency and inverted document frequency and also calculating the measure of similarity between tf and idf finally calculating the cosine similarity between the above states.

\section{REFERENCES}

[1] https://janav.wordpress.com/2013/10/27/tf-idf-and-cosine-similarity/

[2] https://stackoverflow.com/questions/6255835/cosine-similarity-and-tf-idf

[3] https://en.wikipedia.org/wiki/Cosine_similarity

[4] http://www.ics.uci.edu/ djp3/classes/2008_09_26_CS221/Lectures/Lecture26.pdf

[5] http://www.ir-facility.org/scoring-and-ranking-techniques-tf-idf-term-weighting-and-cosine-similarity

[6] http://blog.christianperone.com/2013/09/machine-learning-cosine-similarity-for-vector-space-models-part-iii/

[7] https://courses.cs.washington.edu/courses/cse573/12sp/lectures/17-ir.pdf 